\title{
Comparative Study of Three High Order Schemes for LES of Temporally Evolving Mixing Layers ${ }^{1}$
}

\author{
H.C. Yee ${ }^{a}$, B. Sjögreen ${ }^{b}$, A. Hadjadj; \\ ${ }^{a}$ NASA-Ames Research Center, Moffett Field, CA, 94035, USA \\ ${ }^{b}$ Lawrence Livermore National Laboratory, Livermore, CA, 94551, USA \\ ${ }^{c}$ CORIA UMR 6614 \& INSA de Rouen, 76800 St-Etienne du Rouvray, France
}

\begin{abstract}
Three high order shock-capturing schemes are compared for large eddy simulations (LES) of temporally evolving mixing layers (TML) for different convective Mach numbers $\left(M_{c}\right)$ ranging from the quasi-incompressible regime to highly compressible supersonic regime. The considered high order schemes are fifth-order WENO (WENO5), seventh-order WENO (WENO7) and the associated eighth-order central spatial base scheme with the dissipative portion of WENO7 as a nonlinear post-processing filter step (WENO7fi). This high order nonlinear filter method (H.C. Yee and B. Sjogreen, Proceedings of ICOSAHOM09, June 22-26, 2009, Trondheim, Norway) is designed for accurate and efficient simulations of shock-free compressible turbulence, turbulence with shocklets and turbulence with strong shocks with minimum tuning of scheme parameters. The LES results by WENO7fi using the same scheme parameter agree well with experimental results of Barone et al. (2006), and published direct numerical simulations (DNS) work of Rogers \& Moser (1994) and Pantano \& Sarkar (2002), whereas results by WENO5 and WENO7 compare poorly with experimental data and DNS computations.
\end{abstract}

Key words: High order numerical methods, Numerical methods for turbulence with shocks, DNS, LES, Mixing layer

\section{Introduction}

Part of the inaccuracy in direct numerical simulations (DNS) and large eddy simulations (LES) of turbulent flow using standard high order shock-capturing schemes is due to the fact that this type of computation involves long time integrations. Standard stability and accuracy theories in numerical analysis are not applicable to long time wave propagations and/or long time integrations [38]. The original construction of modern shock-capturing schemes was developed for rapidly developing unsteady shock interactions and short time integrations. Any numerical dissipation inherent in the scheme, even for high resolution shock-

\footnotetext{
${ }^{1}$ This work was performed while the third author was a visiting scholar at the Center for Turbulence Research, Stanford University.
} 
capturing schemes that maintain their high order accuracy in smooth regions (e.g., fifthor seventh-order WENO schemes (WENO5 and WENO7)), will be compounded over long time integration leading to smearing of turbulence fluctuations to un-recognizable forms.

In compressible turbulent combustion/nonequilibrium flows, the constructions of numerical schemes for (a) stable and accurate simulation of turbulence with strong shocks, and (b) obtaining correct propagation speed of discontinuities for stiff reacting terms on "coarse grids" share one important ingredient - minimization of numerical dissipation while maintaining numerical stability. Here "coarse grids" means standard mesh density requirement for accurate simulation of typical non-reacting flows. This dual requirement to achieve both numerical stability and accuracy with zero or minimal use of numerical dissipation is most often conflicting for existing schemes that were designed for non-reacting flows. In addition to the minimization of numerical dissipation while maintaining numerical stability in compressible turbulence with strong shock, Yee \& Sjögreen, Yee and Yee \& Sweby $[46,47,43,42]$ discussed a general framework for the design of such schemes. Yee \& Sjögreen [51], Sjögreen \& Yee [37, 36] and Wei et al. [39, 40] and references cited therein present their recent progress on the subject. In [55], a short overview of this recent progress is given. The discussion addresses three separate yet interwoven types of numerical challenges for high speed turbulent reacting flows containing discontinuities. This paper is confined to the comparison of three specific high order shock-capturing methods on turbulent mixing for non-reacting flows. The study for turbulent mixing for reacting flows is planned.

\section{Recent Progress in Numerical Methods for Turbulence with Strong Shocks}

The current trends in the containment of numerical dissipation in DNS and LES of turbulence with shocks are summarized in Yee \& Sjögreen and Yee et al. [51, 50, 54]. See the cited references for details on these current trends. Before presenting the improved filter schemes and their application to the temporally evolving mixing layers (TML), the key ingredients and the performance of the high order nonlinear filter schemes with preprocessing and post-processing steps in conjunction with the use of a high order nondissipative spatial base scheme $[51,54]$ are briefly illustrated for two test cases.

\subsection{High Order Nonlinear Filter Schemes [34, 49, 51, 54]}

Before the application of a high order non-dissipative spatial base scheme, the preprocessing step to improve stability had split inviscid flux derivatives of the governing equation(s) in the following three ways, depending on the flow types and the desire for rigorous mathematical analysis or physical argument.

- Entropy splitting of Olsson \& Oliger [25] and Yee et al. [45, 46]: The resulting form is non-conservative and the derivation is based on entropy norm stability with numerical boundary closure for the initial value boundary problem. 
- The system form of the Ducros et al. splitting [6]: This is a conservative splitting and the derivation is based on physical arguments.

- Tadmor entropy conservation formulation for systems (Sjögreen \& Yee [35]): The derivation is based on mathematical analysis. It is a generalization of Tadmor's entropy formulation to systems and has not been fully tested on complex flows.

After the application of a non-dissipative high order spatial base scheme on the split form of the governing equation(s), to further improve nonlinear stability from the nondissipative spatial base scheme, the post-processing step of Yee \& Sjögreen [49, 51], Sjögreen \& Yee [34] nonlinearly filtered the solution by a dissipative portion of a high order shock-capturing scheme with a local flow sensor. These flow sensors provide locations and amounts of built-in shock-capturing dissipation that can be further reduced or eliminated. For all the computations shown, the Ducros et al. splitting is employed since a conservative splitting is more appropriate if one does not know if the subject flow is shock-free or turbulence with shocks. Some attributes of the high order filter approach are:

- Spatial Base Scheme: High order and conservative with high order freestream preservation metric evaluation for curvilinear grids. (no flux limiter or Riemann solver)

- Physical Viscosity: Automatically taken into consideration by the base scheme. The same order of central differencing for the viscous derivative as the convective flux derivatives are used.

- Efficiency: One Riemann solve per dimension per time step, independent of time discretizations (less CPU time and fewer grid points than their standard shock-capturing scheme counterparts)

- Accuracy: Containment of numerical dissipation via local wavelet flow sensor

- Well-balanced scheme: These nonlinear filter schemes are well-balanced schemes for certain chemical reacting flows and problem containing geometric source terms [39]

- Stiff Combustion with Discontinuities: For some stiff reacting flow test cases, the high order filter scheme is able to obtain the correct propagation speed of discontinuities whereas the standard high order WENO scheme cannot $[19,56]$.

- Parallel Algorithm: Suitable for most current supercomputer architectures

\subsection{Sample test Cases Illustrating the Efficiency and Accuracy of High Order Filter Schemes}

These filter schemes are efficient, and the total computational cost for a given error tolerance is lower than for standard shock-capturing schemes of the same order. This is of importance, for example, in DNS and in flow control optimization to improve aerodynamic properties, where the flow simulation must be carried out many times during the optimization loop. The efficiency and accuracy of the schemes for a wide variety of flow problems can be found in aforementioned cited references. Here two test cases are illustrated. 


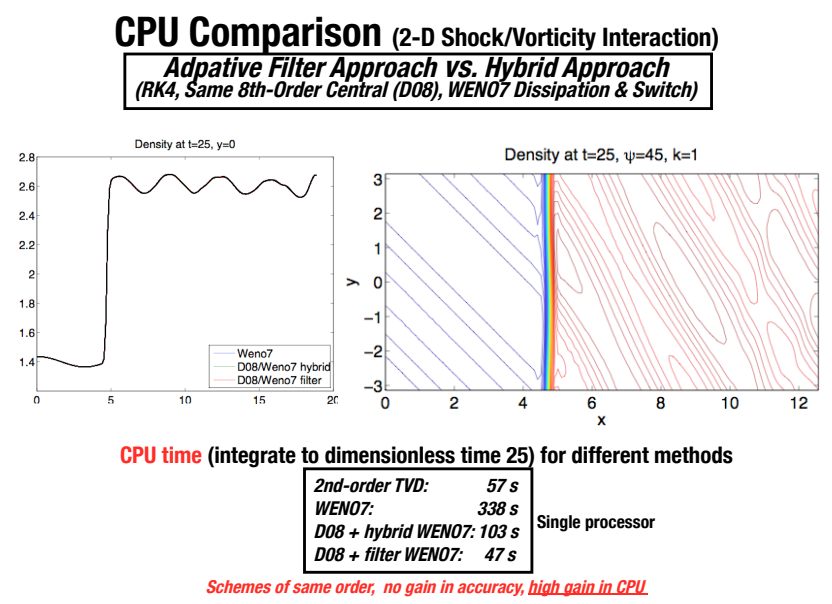

Figure 1: CPU comparison of four shock-capturing schemes.

\subsubsection{D Shock/Vorticity Interaction}

Figure 1 shows a comparison of a second-order TVD, seventh-order WENO (WENO7), hybrid scheme (switch between eighth-order spatial central scheme and WENO7 using wavelet flow sensor as the switch indicator) and the filter scheme WENO7fi (an eighthorder spatial central base scheme and the dissipative portion of WENO7 and the same wavelet flow sensor to guide where the WENO7 dissipation should be applied at the postpossessing nonlinear filter step). A second-order Runge-Kutta method was used for the TVD scheme and the classical fourth-order Runge Kutta method was used for the rest of the spatial scheme. For this particular simple 2D shock-vorticity interaction test case with a simple weak planar shock without structure, WENO7, hybrid, and WENO7fi give the same accuracy. However, there is large gain in CPU time by the filter scheme for this turbulencefree test case. For turbulence with shocks, there is a more beneficial gain both in accuracy and CPU time of the filter schemes over the their standard WENO counterparts.

\subsubsection{D Shock/Turbulence Interaction Problem}

This 1-D compressible inviscid ideal gas problem is one of the most computed test cases in the literature to assess the capability of a shock-capturing scheme in the presence of shock/turbulence interactions. The flow consists of a shock at Mach 3 propagating into a sinusoidal density field with initial data given by $\left(\rho_{L}, u_{L}, p_{L}\right)=$ $(3.857143,2.629369,10.33333)$ to the left of a shock located at $x=-4$, and $\left(\rho_{R}, u_{R}, p_{R}\right)=(1+0.2 \sin (5 x), 0,1)$ to the right of the shock, where $\rho$ is the density, $u$ is the velocity and $p$ is the pressure. The computational domain is $[-5,5]$ and the computation stops at time equal to 1.8. Figure 2 shows the comparison among WENO3, WENO5 and WENO7, and their corresponding filter schemes WENO3fi, WENO5fi and WENO7fi using a very coarse uniform grid of 200 points with the reference solution. The reference solution is obtained with WENO5 using 16000 grid points. WENO5fi required at the most $50 \%$ of the CPU time of WENO5 if third or fourth-order Runge-Kutta time discretization were used. In order for WENO5 to obtain a similar accuracy as WENO5fi, at 


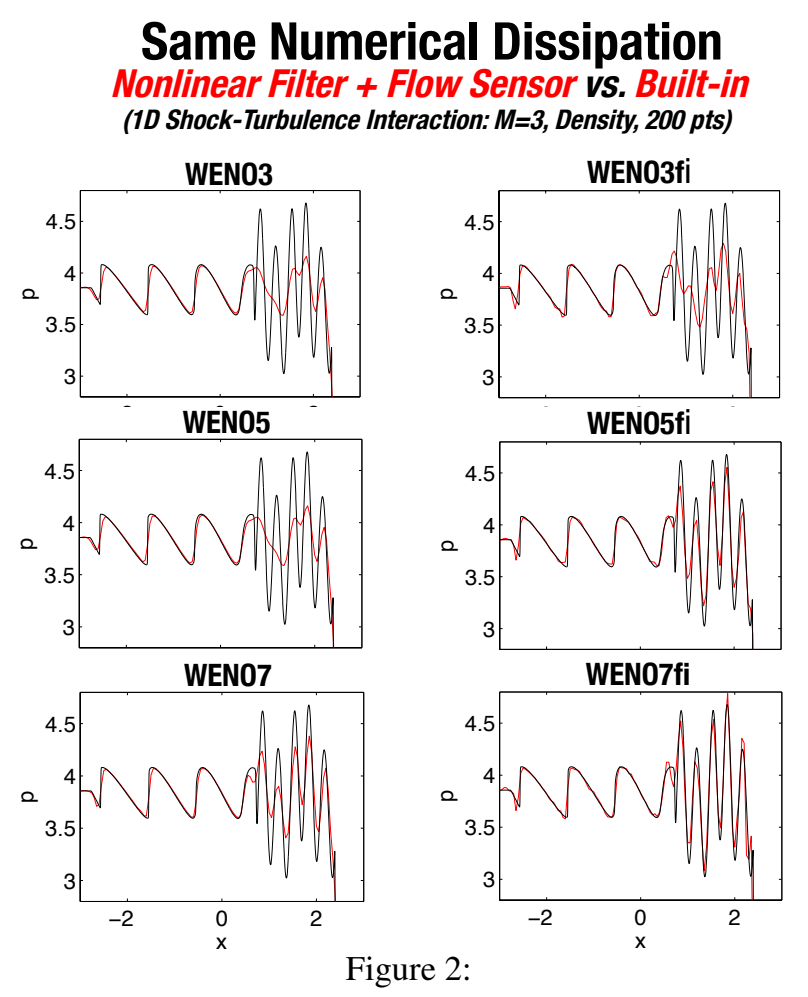

least two times the number of grid points is needed. Moreover, the accuracy of WENO5fi is similar to WENO9 (computation not shown).

\subsection{Objective and Outline}

The objective of this paper is to use the same TML problem setup and convective Mach cases as in [13] to compare the performance of WENO7fi with standard WENO5 and WENO7 for convective Mach $M_{c}=0.1,0.3,0.8,1.0,1.5$. For WENO7fi, no tuning of scheme parameters is needed for all the $M_{c}$ cases. For detailed physics, see [13].

The outline of this paper is as follows: The high order nonlinear filter methods are summarized in Section 2. Recent improvement of the scheme will also be briefly discussed. The problem setup for the temporally resolving mixing layer (TML) is given in Section 3. Numerical results comparing the performance among WENO5, WENO7 and the associated WENO7fi are then presented in Section 4. The comparison is focused only on the momentum thickness and compressibility factor as a function of the studied convective Mach numbers. Even WENO5 is two order lower than WENO7 WENO7fi, it is chosen for the comparison as a benchmark study. This is due to the fact that WENO5 has been widely used for many applications.

\section{Numerical Methods}

This section summarizes the numerical methods to be used for the turbulent TML study. The numerical methods solve the split form of the inviscid flux derivatives according to the 
pre-processing step. The discussion is broken up into two subsections.

\subsection{Original High Order Filter Method}

For turbulence with shocks, instead of solely relying on very high order high-resolution shock-capturing methods for accuracy, the filter schemes [44, 45, 34, 48, 49] take advantage of the effectiveness of the nonlinear dissipation contained in good shock-capturing schemes as stabilizing mechanisms at locations where needed. Such a filter method consists of two steps: a full time step using a spatially high-order non-dissipative base scheme, followed by a post-processing filter step. The post-processing filter step consists of the products of wavelet-based flow sensors and nonlinear numerical dissipations. The flow sensor is used in an adaptive procedure to analyze the computed flow data and indicate the location and type of built-in numerical dissipation that can be eliminated or further reduced. The nonlinear dissipative portion of a high-resolution shock-capturing scheme can be any TVD, MUSCL, ENO, or WENO scheme. By design, the flow sensors, spatial base schemes and nonlinear dissipation models are standalone modules. Therefore, a whole class of low dissipative high order schemes can be derived with ease. Unlike standard shock-capturing and/or hybrid shock-capturing methods, the nonlinear filter method requires one Riemann solve per dimension, independent of time discretizations. The nonlinear filter method is more efficient than its shock-capturing method counterparts employing the same order of the respective methods.

Recently, these filter schemes were proven to be well-balanced schemes [39] in the sense that these schemes preserve exactly certain steady state solutions of the chemical nonequilibrium governing equation. With this added property these filter schemes can better minimize spurious numerics in reacting flows containing mixed steady shocks and unsteady turbulence with shocklet components than standard non-well-balanced shockcapturing schemes. In addition, for some stiff reacting flow test cases, the high order filter scheme is able to obtain the correct propagation speed of discontinuities whereas the standard high order WENO scheme cannot $[19,56]$.

For simplicity of the presentation the discussion for the base scheme and postprocessing step of the filter scheme is restricted to the inviscid part of the Navier-Stokes equations. For viscous gas dynamics the same order of spatial centered base scheme for the convection terms and the viscous terms are employed. For all of the LES computations the classical fourth-order Runge-Kutta time discretization is employed.

Consider the 3-D compressible Euler equations in Cartesian geometry,

$$
U_{t}+\nabla \cdot \mathbf{F}=\mathbf{0} ; \quad U=\left(\begin{array}{c}
\rho \\
\mathbf{m} \\
e
\end{array}\right) ; \mathbf{F}=\left(\begin{array}{c}
\rho \mathbf{u} \\
\rho \mathbf{u} \mathbf{u}^{T}+p \\
\mathbf{u}(e+p)
\end{array}\right)
$$

Here the velocity vector $\mathbf{u}=(u, v, w)^{T}$, the momentum vector $\mathbf{m}=(\rho u, \rho v, \rho w), \rho$ is the density, and $e$ is the total energy. 
In a Cartesian grid denote the grid indices for the three spatial directions as $(j, k, l)$. The spatial base scheme to approximate the $x$ inviscid flux derivatives $F(U)_{x}$ (with the grid indices $k$ and $l$ for the $y$ - and $z$-directions suppressed) is written as, e.g.,

$$
\frac{\partial F}{\partial x} \approx D_{08} F_{j}
$$

where $D_{08}$ is the standard eighth-order accurate centered difference operator. See [35] for the split form of 2.

After the completion of a full Runge-Kutta time step of the base scheme step, the second step is to adaptively apply a post-processing nonlinear filter. The nonlinear filter can be obtained e.g., in the $x$-direction by taking the full seventh-order WENO scheme (WENO7) [16] for the inviscid flux derivative in the $x$-direction and subtracting $D_{08} F_{j}$. The final update of the solution is (with the numerical fluxes in the $y$ - and $z$-directions suppressed as well as their corresponding $y$ - and $z$-directions indices on the $x$ inviscid flux suppressed)

$$
U_{j, k, l}^{n+1}=U_{j, k, l}^{*}-\frac{\Delta t}{\Delta x}\left[H_{j+1 / 2}^{*}-H_{j-1 / 2}^{*}\right] .
$$

The nonlinear filter numerical fluxes usually involve the use of field-by-field approximate Riemann solvers. If the Roe type of approximate Riemann solver [30] is employed, for example, the $x$-filter numerical flux vector $H_{j+1 / 2}$ evaluated at the $U^{*}$ solution from the base scheme step is

$$
H_{j+1 / 2}=R_{j+1 / 2} \bar{H}_{j+1 / 2},
$$

where $R_{j+1 / 2}$ is the matrix of right eigenvectors of the Jacobian of the inviscid flux vector in terms of the Roe's average states. Denote the elements of the vector $\bar{H}_{j+1 / 2}$ by $\bar{h}_{j+1 / 2}^{l}, l=$ $1,2, \ldots, 5$. The nonlinear portion of the filter $\bar{h}_{j+1 / 2}^{l}$ has the form

$$
\bar{h}_{j+1 / 2}^{l}=\frac{\kappa}{2} \omega_{j+1 / 2}^{l} \phi_{j+1 / 2}^{l}
$$

Here $\omega_{j+1 / 2}^{l}$ is the wavelet flow sensor to activate the nonlinear numerical dissipation $\frac{1}{2} \phi_{j+1 / 2}^{l}$ and the original formulation for $\kappa$ is a positive parameter that is less than or equal to one. Some tuning of the parameter $\kappa$ is needed for different flow types. A local $\kappa$ to be discussed next, depending on the local Mach number for low speed flows and depending on local shock strength for high speed flows, would minimize the tuning of parameters. A local flow sensor was discussed by Lo et al. [24] by taking advantge of the Ducros et al. shock flow sensor [7] to obtain a local artificial compression method (ACM) sensor for the original Yee et al. filter scheme [44].

The dissipative portion of the nonlinear filter $\frac{1}{2} \phi_{j+1 / 2}^{l}=g_{j+1 / 2}^{l}-b_{j+1 / 2}^{l}$ is the dissipative portion of, e.g., WENO7 for the local $l$ th-characteristic wave. Here $g_{j+1 / 2}^{l}$ and $b_{j+1 / 2}^{l}$ are numerical fluxes of WENO7 and the eighth-order central scheme for the $l$ th characteristic, respectively. Hereafter, we denote this filter scheme as WENO7fi. 


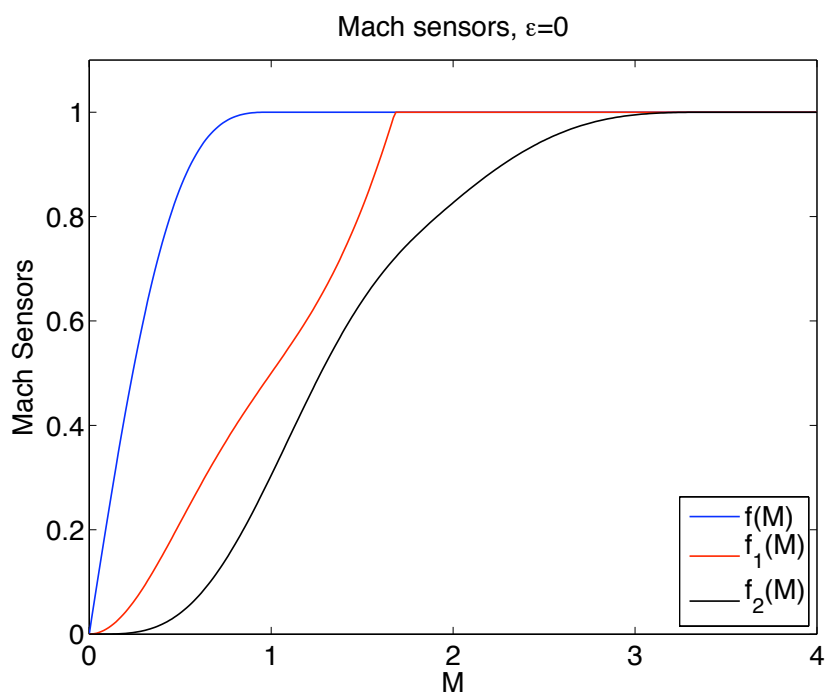

Figure 3: Mach number sensors. $f(M)$ (blue) function by Li and Gu, $f_{1}(M)$ (red) modified $f(M)$, and $f_{2}(M)$ (black) (includes low supersonic Mach numbers).

A summary of the three basic steps to obtain $\omega_{j+1 / 2}^{l}$ can be found in $[34,49]$. For example, the flow sensor $\omega_{j+1 / 2}^{l}$ to activate the shock-capturing dissipation using the cut off procedure is a vector (if applied dimension-by-dimension) consisting of "1's" and " $\mathbf{0}$ 's". For all of the computations, a three-level second-order Harten multiresolution wavelet decomposition of the computed density and pressure is used as the flow sensor [34].

\subsection{Improved High Order Filter Method}

Previous numerical experiments on a wide range of flow conditions [44, 45, 34, 48, 49] indicated that the original filter scheme improves the overall accuracy of the computation compared with standard shock-capturing schemes of the same order. Studies found that the improved accuracy is more pronounced if the parameter $\kappa$ in (4) is tuned according to the flow type locally. For hypersonic flows with strong shocks, $\kappa$ is set to 1 . For high subsonic and supersonic flows with strong shocks, $\kappa$ is in the range of $(0.3,0.9)$. For low speed turbulent flows without shocks or long time integration of smooth flows, $\kappa$ can be one to two orders of magnitude smaller than 1 . In other words, $\kappa$ should be flow location, shock strength and local flow type dependent. The improved $\kappa$ proposed in [51] consists of a simple global $\kappa$ for smooth flows and a local $\kappa$ for problems with shocks and turbulence.

\subsubsection{An efficient global $\kappa$ for low Mach number and smooth flows:}

The flow speed indicator formula of $\mathrm{Li} \& \mathrm{Gu}$ to overcome the shortcomings of "low speed Roe scheme" [23] was modified to obtain an improved global $\kappa$ denoted by $\bar{\kappa}$ for (4) to minimize the tuning of the original $\kappa$ for low Mach number flows. $\bar{\kappa}$ has the form: 


$$
\bar{\kappa}=f_{1}(M) \kappa,
$$

with

$$
f_{1}(M)=\min \left(\frac{M^{2}}{2} \frac{\sqrt{4+\left(1-M^{2}\right)^{2}}}{1+M^{2}}, 1\right) .
$$

Here $M$ is the maximum Mach number of the entire computational domain at each stage of the time evolution. $f_{1}(M)$ has the same form as [23] except there is an extra factor " $\frac{M}{2}$ ", added to the first argument on the right-hand-side of the original form $f(M)$ in equation (18) of [23]. The added factor provides a similar value of the tuning $\kappa$ observed from numerical experimentation reported in aforementioned cited references. With the flow speed indicator $f_{1}(M)$ in front of $\kappa$, the same $\kappa$ used for the supersonic shock problem can be used without any tuning for the very low speed turbulent flow cases. Another minor modification of the above is,

$$
\overline{f_{1}(M)}=\max \left(\min \left(\frac{M^{2}}{2} \frac{\sqrt{4+\left(1-M^{2}\right)^{2}}}{1+M^{2}}, 1\right), \epsilon\right),
$$

where $\epsilon$ is a small threshold value to avoid completely switching off the dissipation. A function which retains the majority of $f_{1}(M)$ but includes larger Mach number for not very strong shocks is

$$
f_{2}(M)=(Q(M, 2)+Q(M, 3.5)) / 2
$$

or

$$
\overline{f_{2}(M)}=\max ((Q(M, 2)+Q(M, 3.5)) / 2, \epsilon)
$$

where

$$
Q(M, a)=\left\{\begin{array}{ll}
P(M / a) & M<a \\
1 & \text { otherwise }
\end{array} .\right.
$$

The polynomial

$$
P(x)=x^{4}\left(35-84 x+70 x^{2}-20 x^{3}\right)
$$

is monotonically increasing from $P(0)=0$ to $P(1)=1$ and has the property that $P^{\prime}(x)$ has three continuous derivatives at $x=0$ and at $x=1$.

Below supersonic speeds, a simple and efficient global $\bar{\kappa}$ can be obtained according to the maximum Mach number of the entire flow field and the value is determined by $f_{1}(M)$ or $f_{2}(M)$ for non-zero $\omega_{j+1 / 2}^{l}$. It is noted that if the original $f(M)$ were used instead of $f_{1}(M)$ or $f_{2}(M)$ in Eq.(5), the amount of nonlinear filter dissipation could be too large for very low speed turbulent flows (for the same fixed $\kappa$ ). See Fig. 3 for details. 


\subsubsection{Local flow sensor for a wide spectrum of flow speed and shock strength}

At each time step and grid point, the aforementioned global $\bar{\kappa}$ is not sufficient to reduce the amount of numerical dissipation where needed for flows that contains a variety of flow features. A more appropriate approach is to obtain a"local $\kappa$ " that is determined according to the above at each grid point. If known, a dominating shock jump variable should be used for shock detections. In other words, the filter numerical flux indicated in Eq.(4) is replaced by:

$$
\bar{h}_{j+1 / 2}^{l}=\frac{1}{2}\left[\kappa_{j+1 / 2}^{l} \omega_{j+1 / 2}^{l} \phi_{j+1 / 2}^{l}\right] .
$$

In the case of unknown physics and without experimental data or theory for comparison, $\kappa_{j+1 / 2}^{l}$ has to depend on the local Mach number in low speed or smooth flow regions, depend on local shock strength in shock regions and depend on turbulent fluctuations in vortical regions in order to minimize the tuning of parameters. According to the flow type locally, for each non-zero wavelet indicator $\omega_{j+1 / 2}^{l}, \kappa_{j+1 / 2}^{l}$ should provide the aforementioned amount (between $(0,1)$ ) to be filtered by the shock-capturing dissipation $\phi_{j+1 / 2}^{l}$. For problems containing turbulence and strong shocks, the shock strength should come into play. One measure of the shock strength can be based on the numerical Schlieren formula [12] for the chosen variables that exhibit the strongest shock strength. In the vicinity of turbulent fluctuation locations, $\kappa_{j+1 / 2}^{l}$ will be kept to the same order as in the nearly incompressible case, except in the vicinity of high shear and shocklets.

Due to the fact that $\bar{\kappa}$ works well for local Mach number below $0.4, \kappa$ only needs to be modified in regions that are above 0.4 . In other words, the final value of $\kappa_{j+1 / 2}^{l}$ is determined by the previous local $\bar{\kappa}$, if the local Mach number is below 0.4. If the local Mach number is above 0.4 , at discontinuities detected by the non-zero wavelet indicator $\omega_{j+1 / 2}^{l}, \kappa_{j+1 / 2}^{l}$ is determined by the shock strength (normalized between $(0,1)$ ) based on the Schlieren formula near discontinuities. Again, if known, dominating shock jump variables should be used for shock detections. At locations with turbulence, determined by the turbulent sensor (e.g., $\omega_{j+1 / 2}^{l}$ obtained from employing wavelets with higher order vanishing moments), $\kappa_{j+1 / 2}^{l}$ is kept to the same order as in the nearly incompressible case, except in the vicinity of high shear and shocklet locations, where a slightly larger $\kappa_{j+1 / 2}^{l}$ would be used. Methods in detecting turbulent flow can be (a) Wavelets with higher order vanishing moments, and (b) Wavelet based Coherent Vortex Extraction (CVE) of Farge et al. [8] (Split the flow into two parts: Active coherent vortices and incoherent background flow).

Results by the local $\kappa_{j+1 / 2}^{l}$ that take the local flow speed and shock strength into consideration will be reported in [53], an expanded version of [51]. Preliminary study with more complex shock turbulence problems and the applicability of even wider flow types indicates the necessity of the local $\kappa_{j+1 / 2}^{l}$.

In this paper, all the computations use the global $\bar{\kappa}$, the Ducros et al. splitting of the inviscid flux derivatives and WENO7fi using the global $\bar{\kappa}$ in conjunction with the classical fourth-order Runge-Kutta temporal discretization. $\kappa=0.7$ for all test cases. 


\section{Description of the Physical Problem and Computational Setup}

LES using the subgrid model of [27] of a TML between two streams with equal and opposite velocities was considered in [13]. In [13] the three main characteristics of compressible TML (the self similarity property, compressibility effects and the presence of large-scale structure with shocklets for high $M_{c}$ ) were considered for the LES study. The role of compressibility in turbulent mixing layers remains an important issue in aeronautics. For example, in the design of high-speed (supersonic or hypersonic) propulsion devices, the stabilizing effect of compressibility may reduce the efficiency of engines in mixing the fuel and the oxidizer. One of the objectives of the study in [13] is to use WENO7fi to investigate the compressibility effects in highly sheared turbulent flows subjected to strong shocks. Here WENO7fi refers to the pre-processing step (Ducros et al. splitting of the inviscid flux derivative) in conjunction with the eighth-order central spatial base scheme with the dissipative portion of WENO7 and the global flow sensor discussed in Section 2 as the post-processing nonlinear filter step. The objective of the current investigation is to compare the performance among WENO7fi, WENO5 and WENO7 using the same problem setup, computational domain and grid size as in [13].

\subsection{TML Problem Setup in [13]}

The configuration of the TML is shown in Fig.4. Five test cases are carried out with different convective Mach numbers $\left(M_{c}=0.1,0.3,0.8,1.0\right.$ and 1.5) ranging from the incompressible case $M_{c}=0.1$ up to the supersonic case $M_{c}=1.5$. The $M_{c}=1.5$ case correspond to a highly compressible mixing layer, whereas the first two cases $M_{c}=0.1$ and $M_{c}=0.3$ can be considered as quasi-incompressible and are used to compare with the experimental results of an incompressible shear layer. All of the simulations described below are performed at an initial Reynolds number, $R e_{\omega_{0}}$, based on the mean velocity difference $\Delta U=2 U_{1}=-2 U_{2}$, the average viscosity of the free streams and the vorticity thickness $\delta_{\omega_{0}}$ of 800 with $\delta_{w_{0}}=4 \delta_{\theta_{0}}$, where $\delta_{\omega}=\Delta U /\langle\partial u / \partial y\rangle_{\max }$ is the vorticity thickness of the shear layer, and $\delta_{\theta}$ is the momentum thickness (see [13] for details). The values of $R e_{\omega}$ can be as large as $3 \times 10^{5}$ at the end of the simulation, which is one order of magnitude higher than similar DNS and LES computations reported in the literature [28, 26, 9]. The mean flow is initialized with a tangent hyperbolic profile for the streamwise velocity, $u(y)=\frac{1}{2} \Delta U \tanh \left[y /\left(2 \delta_{\theta_{0}}\right)\right]$, while the two other velocity components are set to zero. In addition to these mean values, three-dimensional turbulent fluctuations $\left(u^{\prime}, v^{\prime}, w^{\prime}\right)$ are imposed, while initial pressure and density are set constant. Since the simulation is temporal, the initial velocity perturbations are computed using a digital filter technique [18]. This procedure utilizes the prescribed Reynolds stress tensor and length scales of the problem concerned to generate the corresponding fluctuating velocity field, taking into account the nature of the autocorrelation function for the prevailing turbulence. See [13] for details. The digital filter algorithm is given in Appendix B of [13]. The length scales are chosen as $\delta_{w_{0}}$ in each direction. The Reynolds stresses have a Gaussian shape in $y$ with amplitudes chosen to be similar to the experimental peak intensities observed in incompressible mixing layer [2]. 


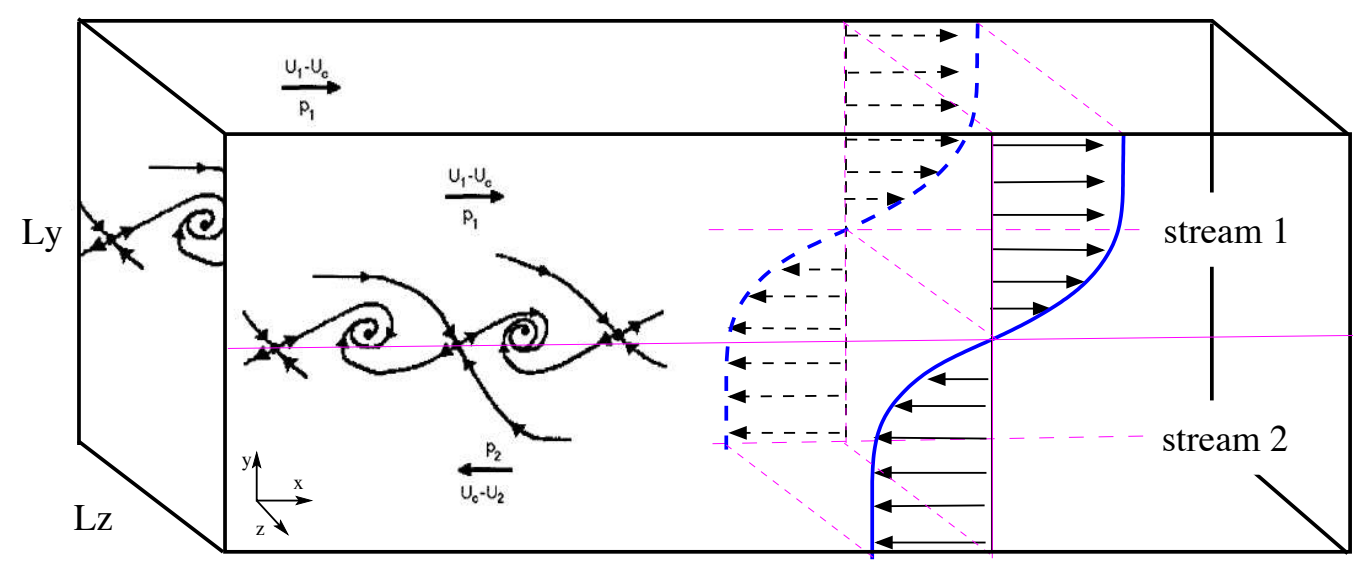

Lx

Figure 4: Schematic configuration of temporal mixing layer.

Periodic boundary conditions are enforced in the streamwise $(x)$ and spanwise $(z)$ directions, while non-reflecting conditions are applied at both top and bottom boundaries ( $y$ direction). The use of a periodic boundary condition in the $x$ direction corresponds to the temporal formulation of mixing layer evolution, which evolves only in time as it spreads in $y$.

\subsection{Mesh Requirements}

Similar to [9], a computational domain of lengths $L_{x} \times L_{y} \times L_{z}=1200 \delta_{\theta_{0}} \times 370 \delta_{\theta_{0}} \times$ $270 \delta_{\theta_{0}}$ is used with the corresponding mesh points $N_{x} \times N_{y} \times N_{z}=512 \times 211 \times 131$. The same grid system uniformly spaced in the $x$ and $z$ directions and stretched in the $y$ direction is employed for all considered cases. The stretching function for the $y$-direction is based on $\frac{L_{y}}{2} \frac{\sinh \left(b_{y} \eta\right)}{\sinh b_{y}}$, where $L_{y}$ is the box size in the $y$-direction and the stretching factor $b_{y}=3.4$. The mapped coordinate $\eta$ is equally spaced and runs from -1 to 1 . The grid used in this study contains an order of magnitude fewer cells than that of the DNS of Pantano and Sarkar [28] compared to the domain length. To ensure that the computational domain in the $x$ - and $z$-directions is sufficiently wide, the two-point correlation functions were analyzed in [13].

\section{Numerical Results}

LES using the subgrid model of [27] and WENO7fi was performed in [13] on the TML problems at different convective Mach numbers $M_{c}(0.1,0.3,0.8,1.0,1.5)$. Studies in [13] show the level of good agreement obtained between LES and DNS for convective Mach number 0.3. In higher convective Mach numbers (up to 1.5), LES results are in good agreement with the experiments. Also, the principal compressibility effects such as the reduction of the spreading rate and the turbulence intensities are well predicted. 

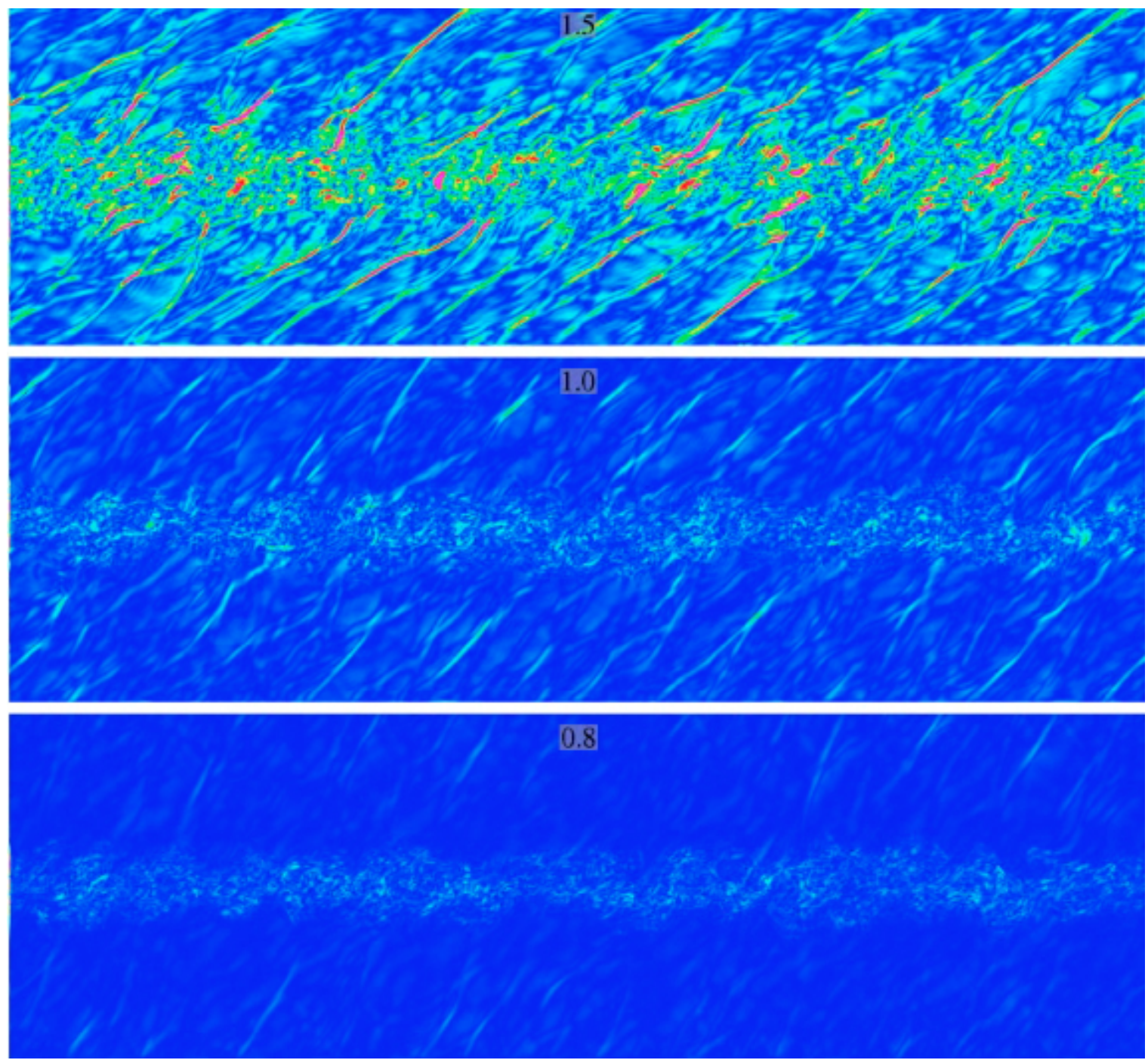

Figure 5: 2-D cut at midplane of instantaneous dilatation flow-field at $\tau=1000$ for three different convective Mach numbers ( $M_{c}=1.5$ (top), 1.0 (middle), and 0.8 (bottom)). 
The flow features of this TML are determined by $M_{c}$. LES computations are carried out up to dimensionless time $\tau=t \Delta U / \delta_{\theta_{0}} \simeq 3000$ for the higher convective Mach number cases and $\tau \simeq 1200$ for the quasi-incompressible cases. Shocklets developed for $M_{c} \geq 0.8$. The shocklets are stronger and more complicated as $M_{c}$ increases. Figure 4 shows the 2-D cut at midplane of instantaneous dilatation flow field at $\tau=1000$ for three different convective Mach numbers $\left(M_{c}=1.5,1.0\right.$, and 0.8$)$. Figure 4 indicates the different flow pattern as a function of $M_{c}$. Figure 5 shows the 2-D cut at mid plane and 3-D of instantaneous numerical Schlieren of vorticity at $\tau=2000$ and $M_{c}=1.5$ by WENO7fi. Figure 6 shows 3-D iso-surfaces of instantaneous vorticity field from different viewing angles for $M_{c}=1.5$.

For each $M_{c}$, after a transient phase, the momentum thickness approaches a separate linear growth regime. LES results by WENO7fi for different convective Mach numbers agree well with the analytically predicted slopes whereas WENO5 and WENO7 do not agree well with the predicted slope for most of the $M_{c}$ cases.

Figures 7-11 show the momentum thickness comparison among the three schemes for each of the $M_{c}$ cases. It is interesting to note that the global $\bar{\kappa}$ used by WENO7fi only shows a slight improvement over WENO5 and WENO7 for $M_{c}=0.8$ and 1.0. For the compressibility factor computations to be shown next, WENO7fi compares well with experimental data for all studied $M_{c}$, whereas this is not the case for WENO5 and WENO7.

Figure 12 displays the compressibility factor as a function of $M_{c}$ by three high order schemes comparing with published work and experiments. This figure shows the superior performance of WENO7fi compared with WENO5 and WENO7. The LES results using WENO7fi agree well with experimental results of Barone et al. (2006) [1], and published direct numerical simulations (DNS) work of Rogers \& Moser (1994) [31] and Pantano \& Sarkar (2002) [28]. In all $M_{c}$ cases, no tuning of WENO7fi scheme parameters was needed. For all the $M_{c}$ cases considered, solutions by WENO5 and WENO7 compared poorly with experimental data and DNS computations.

The unsteady time evolution of turbulence with shocklets for $M_{c}=0.8,1.0$ and 1.5 among WENO5, WENO7 and WENO7fi are very different in terms of the location and strength of the shocklets and turbulent fluctuation pattern. Figure 13 shows the 2-D cut at midplane of the numerical Schlieren of vorticity at $\tau=2000$ and $M_{c}=1.5$ computed by WENO7fi and WENO5. Figure 14 shows the 2-D cut at midplane of instantaneous dilatation flow-field at $\tau=2000$ and $M_{c}=1.5$ computed by WENO7fi and WENO5.

\section{Concluding Remarks}

The present work serves as a validation and performance study of the improved filter schemes of [51] on a representative complex compressible turbulent flow consisting of a wide range of flow speeds. All the computations use the global $\bar{\kappa}$, the Ducros et al. splitting of the inviscid flux derivatives and WENO7fi with $\kappa=0.7$ described in Section 2.2.1. In all $M_{c}$ cases, no tuning of WENO7fi scheme parameters were needed. Studies indicated that WENO7fi compared well with experimental data and published DNS work. For all the $M_{c}$ 

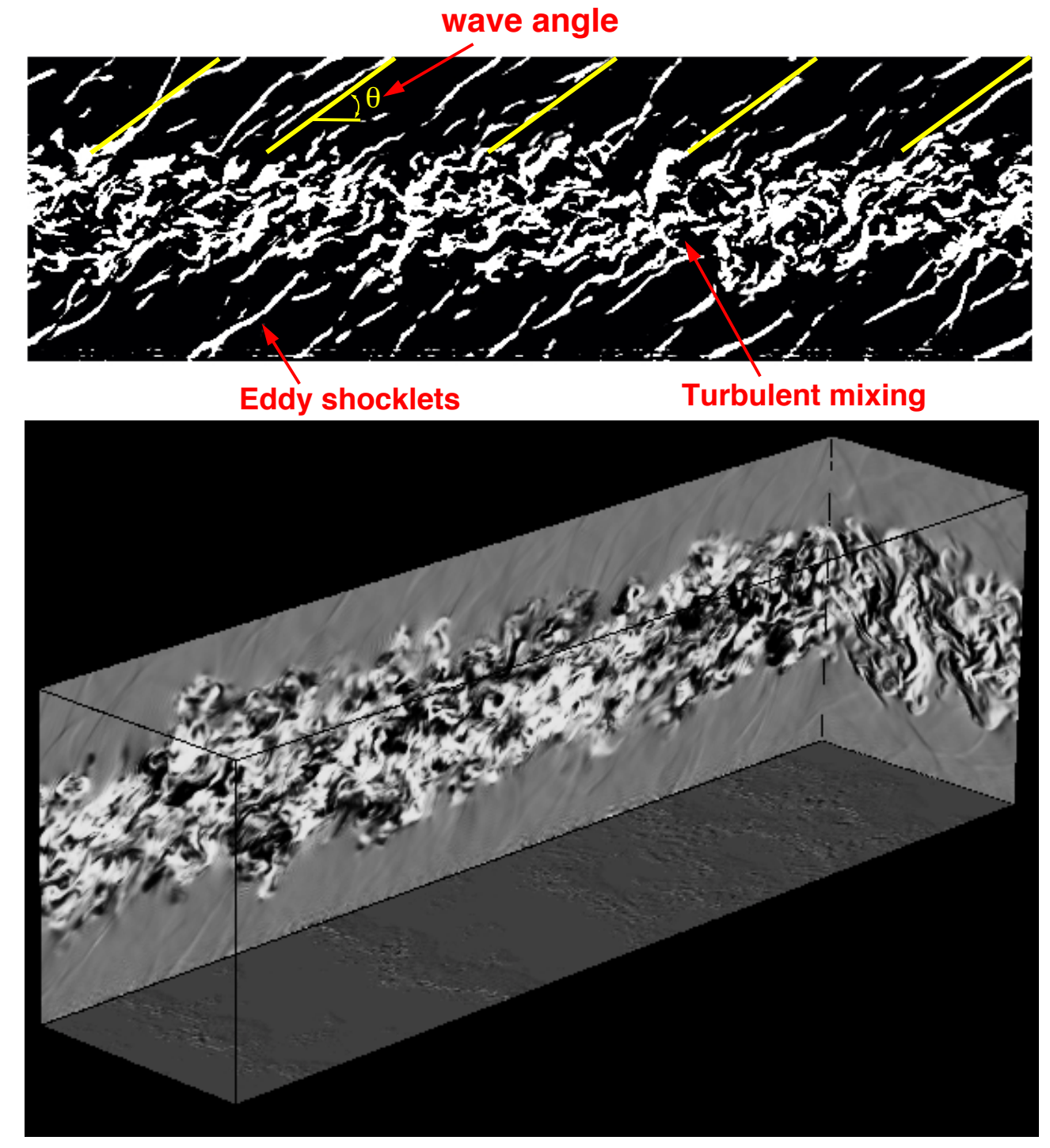

Figure 6: Instantaneous numerical Schlieren pictures at $\tau=2000$ and $M_{c}=1.5$ by WENO7fi indicating shocklets formation. 


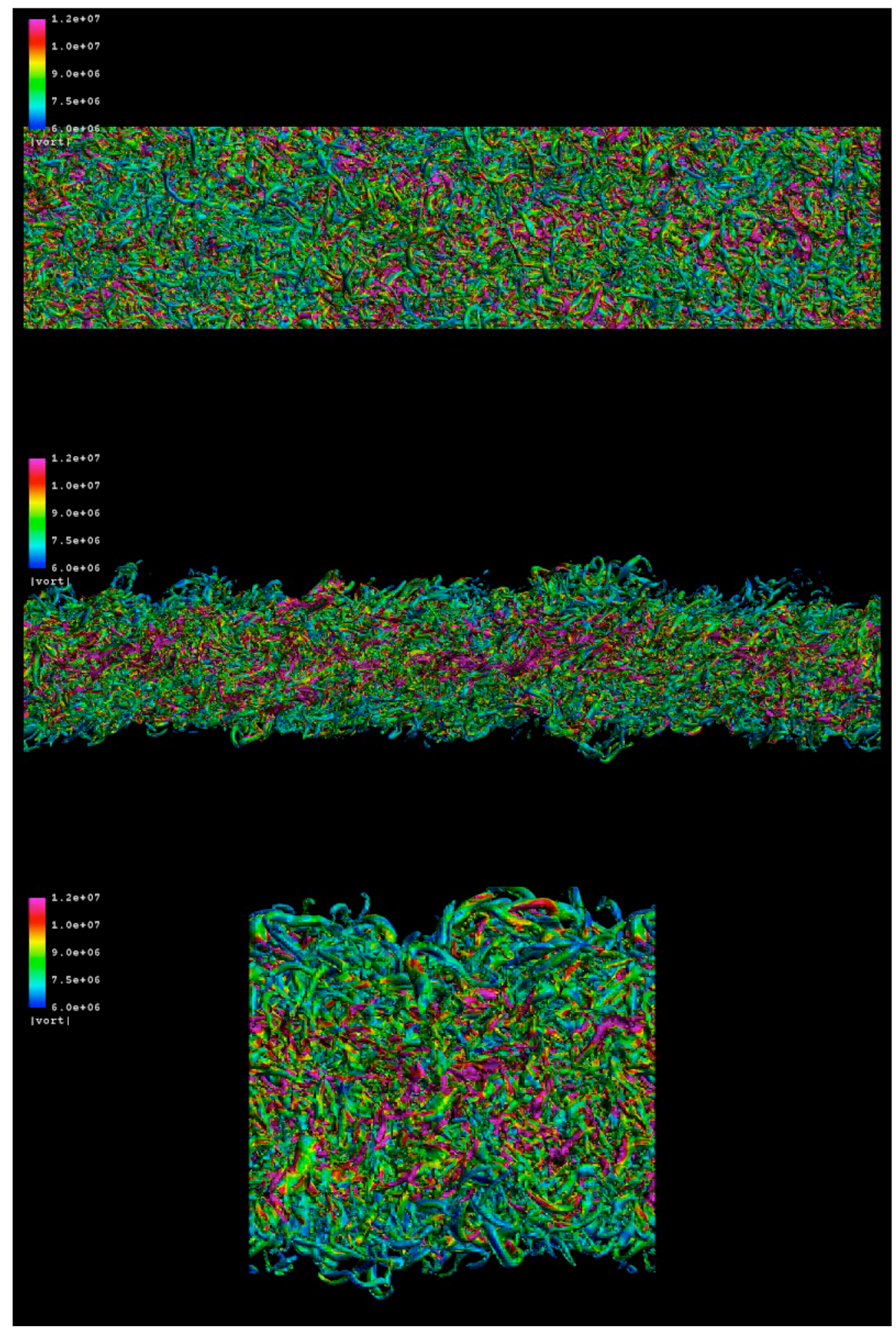

Figure 7: Temporal mixing: Instantaneous numerical Schlieren of vorticity by WENO7fi at $\tau=2000, M_{c}=$ 1.5 , top and side views. 


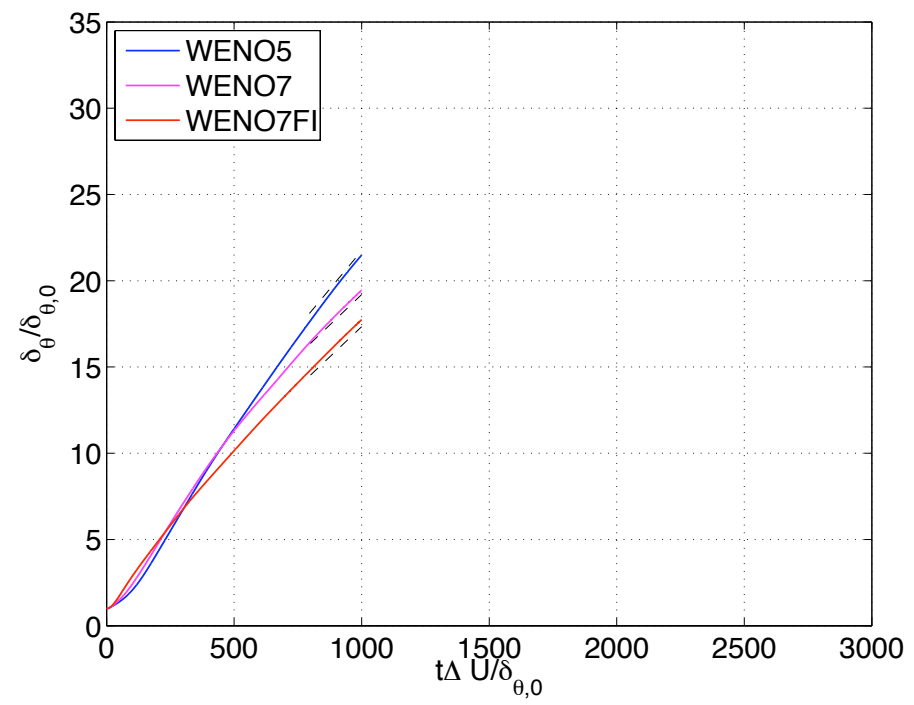

Figure 8: Temporal mixing: Momentum thickness comparison for $M_{c}=0.1$

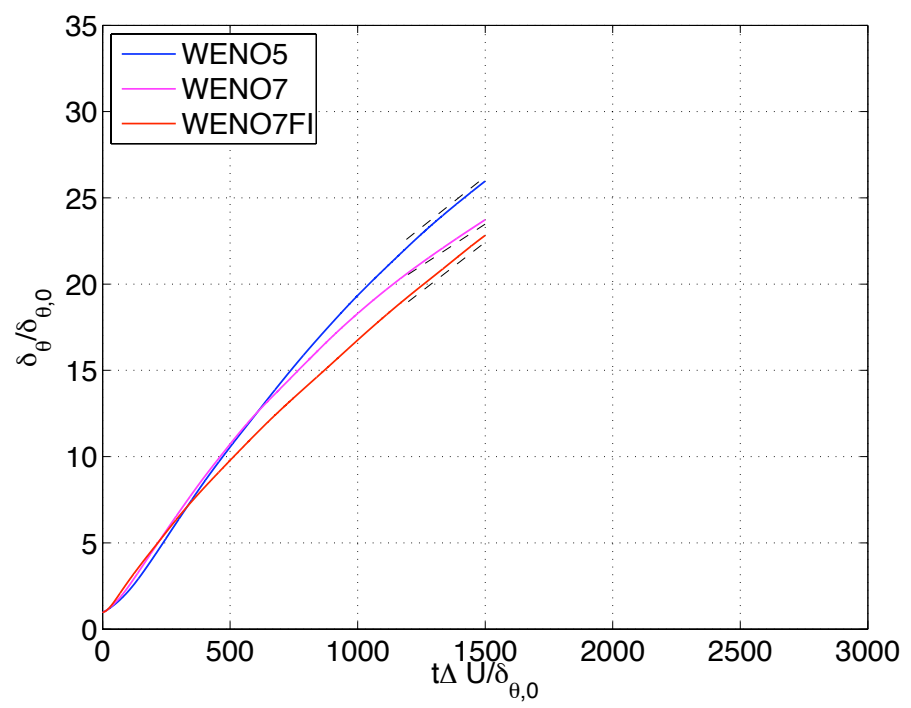

Figure 9: Temporal mixing: Momentum thickness comparison for $M_{c}=0.3$ 


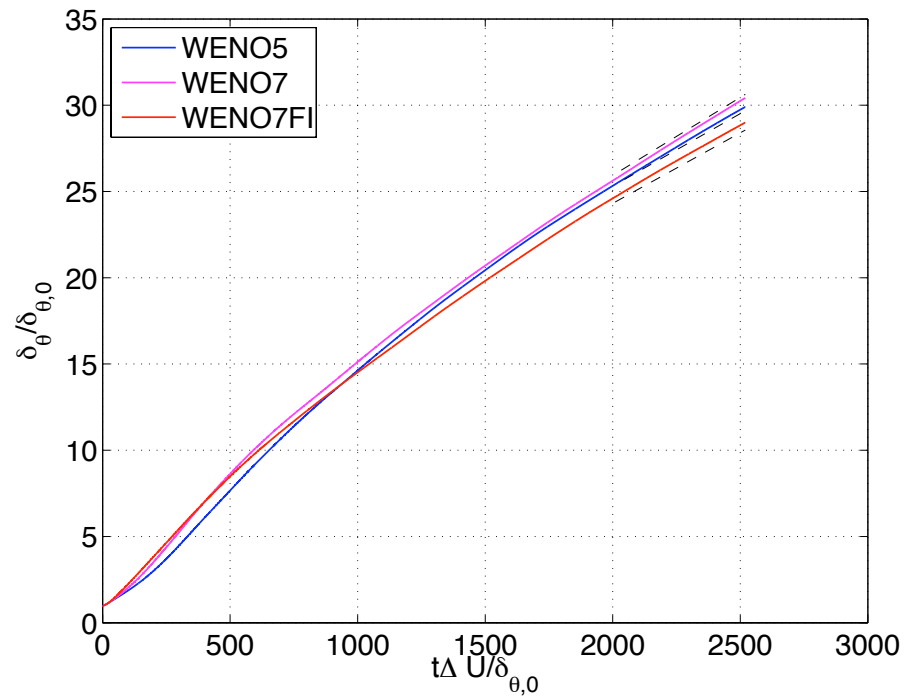

Figure 10: Temporal mixing: Momentum thickness comparison for $M_{c}=0.8$

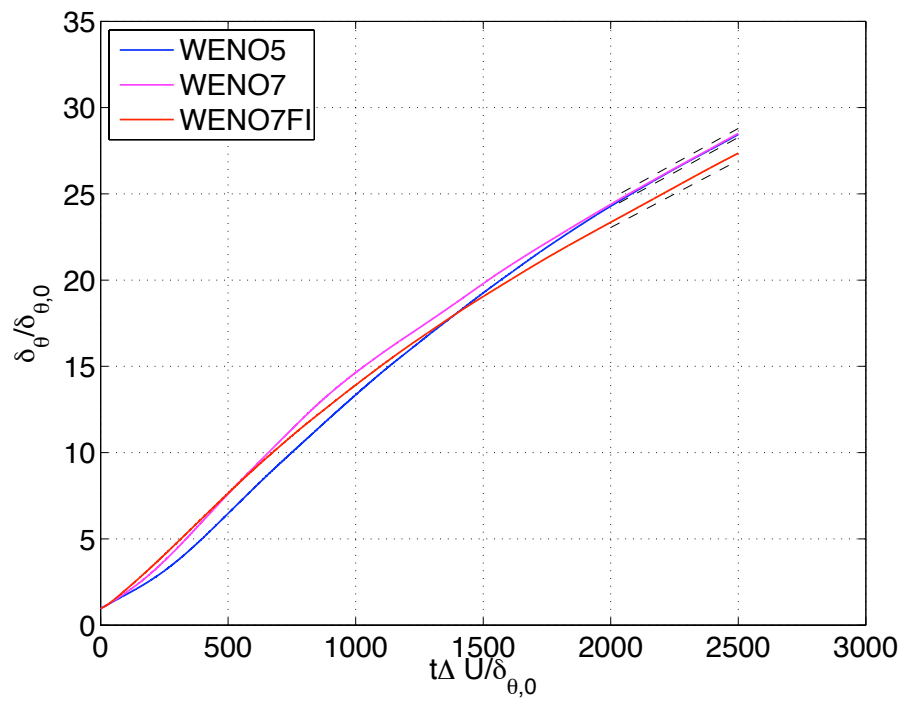

Figure 11: Temporal mixing: Momentum thickness comparison for $M_{c}=1.0$ 


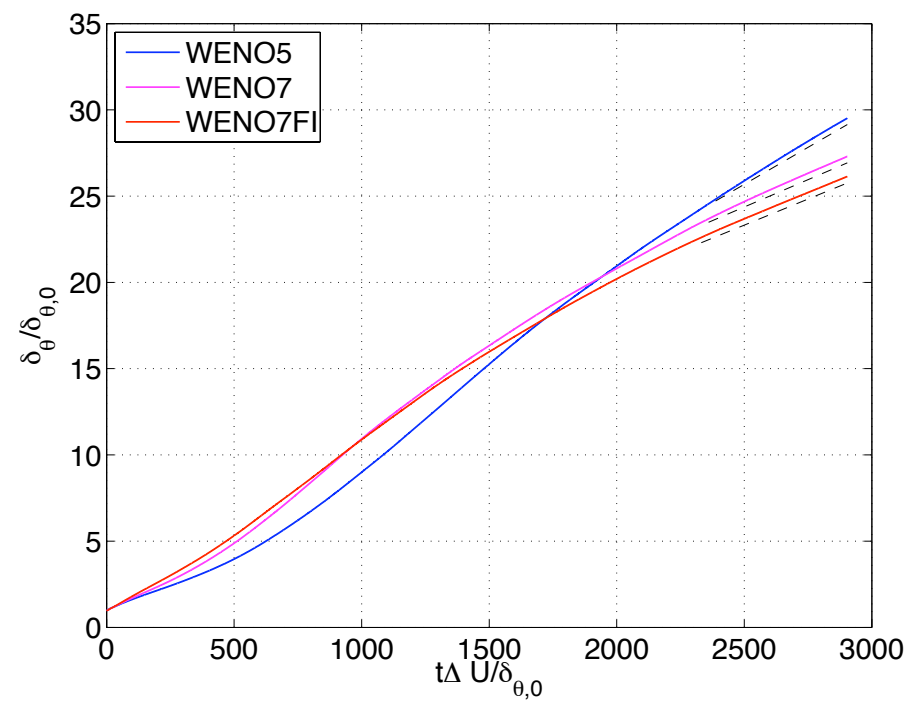

Figure 12: Temporal mixing: Momentum thickness comparison for $M_{c}=1.5$

cases considered, solutions by WENO5 and WENO7 compared poorly with experimental data and DNS computations.

The same high order filter scheme is being used for the simulation of two much higher $M_{c}$ cases of $M_{c}=2,3$. The computational box size, especially in the $y$-direction has to be doubled or more. A finer grid is also needed in order to obtain an accurate and stable solution. These computations are many times more CPU-intensive than the lower $M_{c}$ cases. Results will be reported in a forthcoming paper.

\section{Acknowledgments}

The authors wish to express their gratitude to T. Sandstrom and C. Henze of the Visualization Group, and A. Lazanoff and J. Chang of the Scientific Consultant Group, Code TN, NASA Ames for their help. Special thanks to M. Rogers and A. Wray for their valuable discussion during the course of this research. The support of the DOE/SciDAC SAP grant DE-AI02-06ER25796 is acknowledged. Part of the work by the first author was performed under the NASA Fundamental Aeronautics Hypersonic Program. Work by the second author was performed under the auspices of the U.S. Department of Energy by Lawrence Livermore National Laboratory under Contract DE-AC52-07NA27344.

\section{References}

[1] M.F. Barone, W.L. Oberkampf and F.G. Blottner, Validation Case Study: Prediction of Compressible Turbulent Mixing Layer Growth Rate, AIAA J., 44 (2006) 1488-1497. 


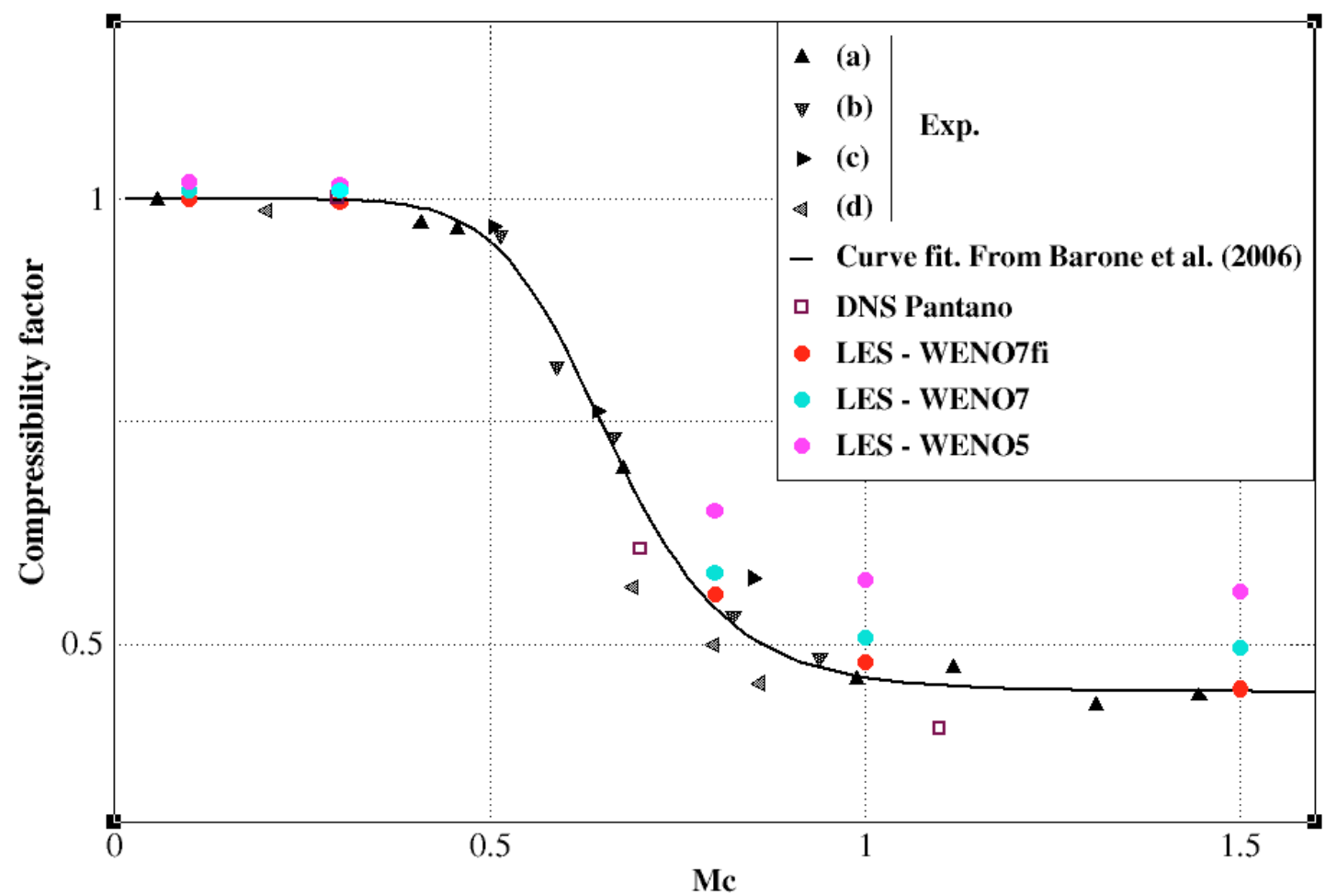

Figure 13: Temporal mixing: Compressibility factor as function of the convective Mach number from different experimental mixing-layer studies selected by Barone et al. [1]: (a) Bogdanoff [4]; Papamoschou and Roshko [29]; (b) Chinzei at al. [5]; (c) Samimy and Elliott [32, 33]; — nonlinear regression curve from [1] with $\Phi\left(M_{c}\right)=1-a_{1}\left[1-1 /\left(1+a_{2} M_{c}^{a_{3}}\right)\right], a_{1}=0.5537, a_{2}=31.79, a_{3}=8.426$. (d) Gruber et al. [11]. Comparison among LES computations by WENO7fi (red solid circles); WENO5 (magenta) and WENO7 (blue) for $M_{c}=0.1,0.3,0.8,1.0,1.5$. 
LES, Mc=1.5, $1-\tanh [\mathrm{k} * \operatorname{div} \mathrm{U} /$ /div Ulmax $]$, WENO7fi vs WENO5
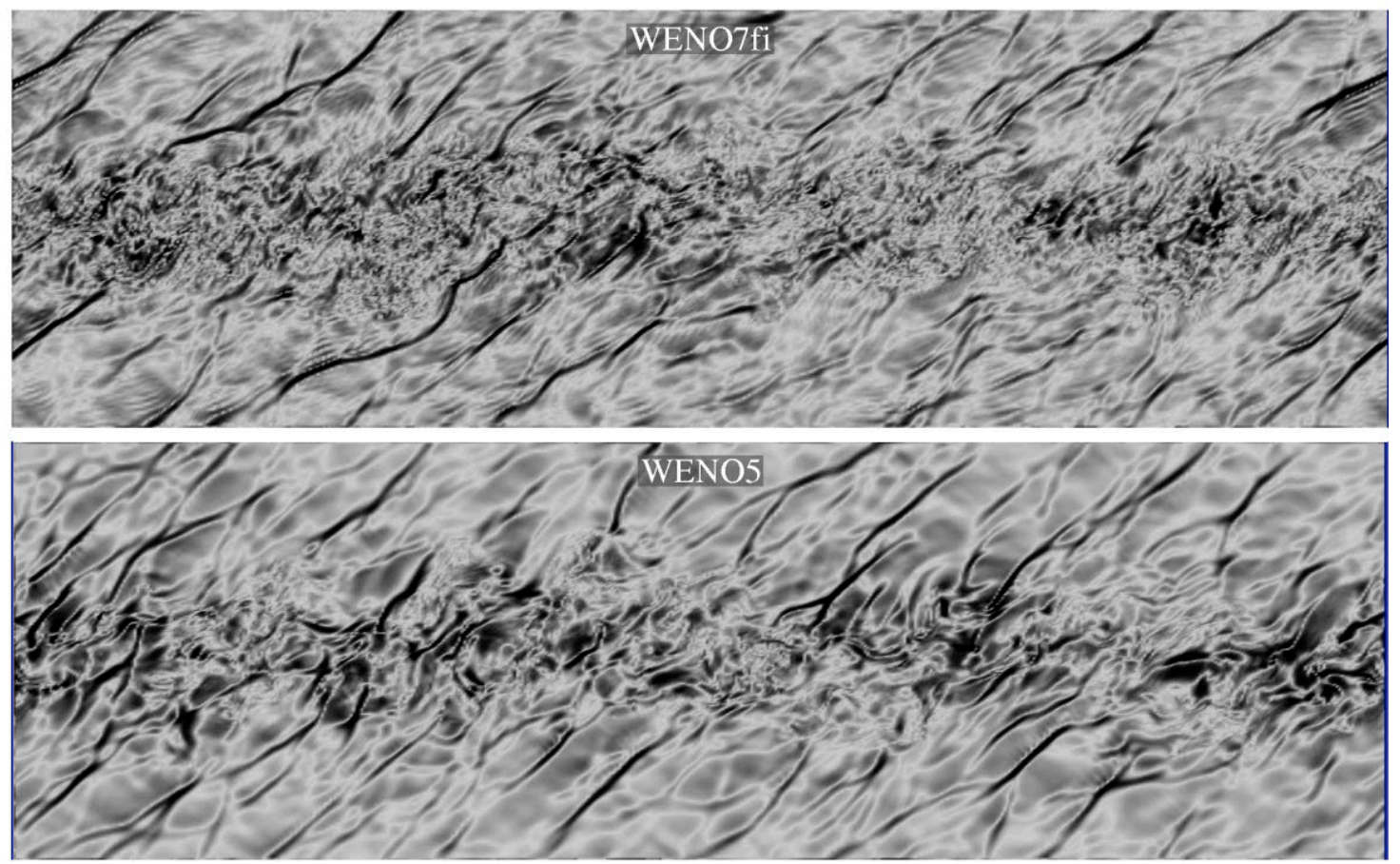

Figure 14: Temporal mixing: 2-D cut at mid plane of instantaneous numerical Schlieren of vorticity at $\tau=$ 2000, $M_{c}=1.5$. Top (WENO7fi), bottom (WENO5). 
LES, Mc $=1.5$, scalar=ldiv Vl, WENO7fi vs WENO5
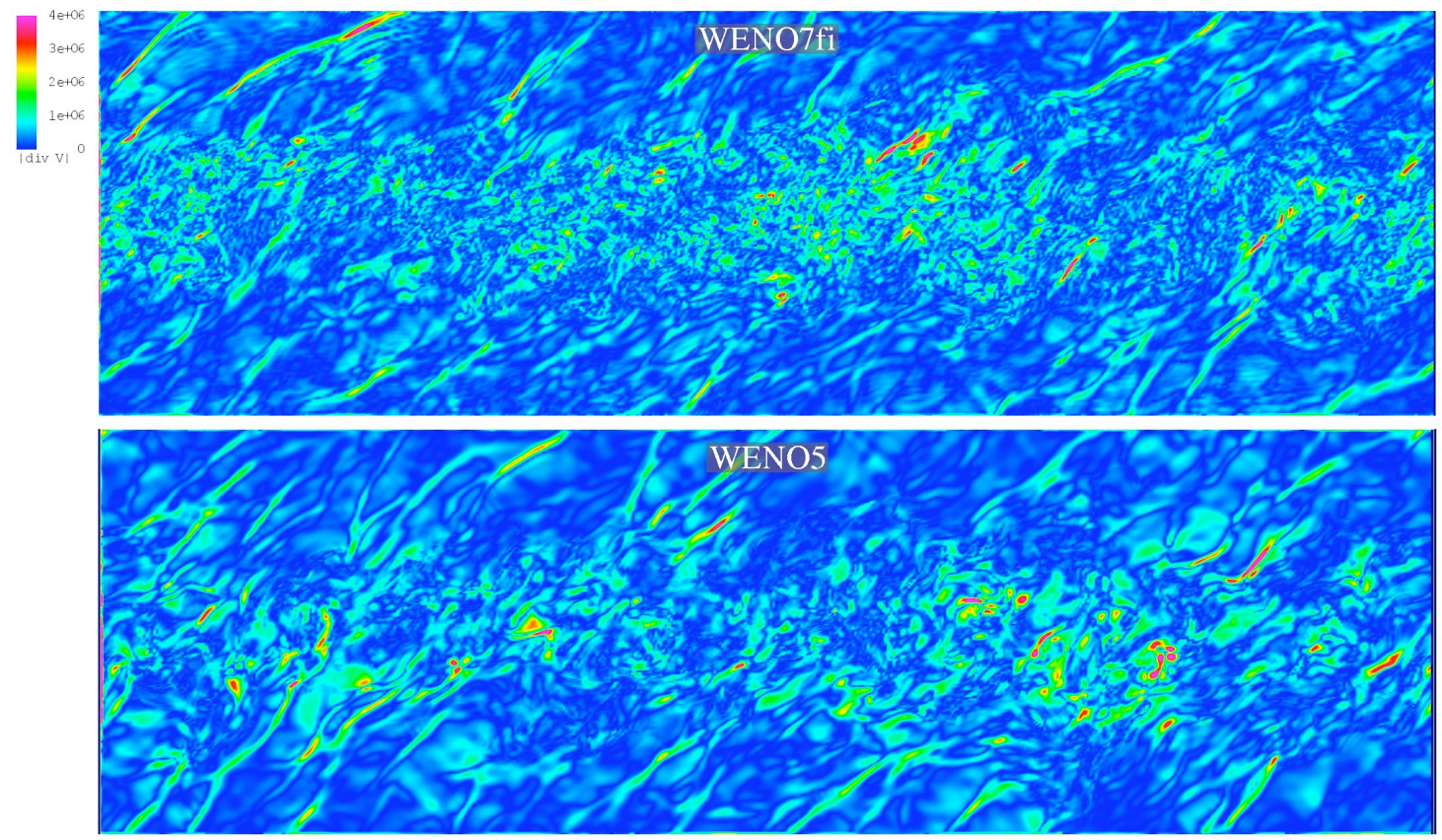

Figure 15: Temporal mixing: 2-D cut at mid plane of instantaneous dilatation flow-field at $\tau=2000, M_{c}=$ 1.5. Top (WENO7fi), bottom (WENO5). 
[2] J. H. Bell and R. D. Mehta, Development of a Two-Stream Mixing Layer from Tripped and Untripped Boundary Layers, AIAA J., 2812 (1990) 2034-2042.

[3] A. Bhagatwala and S.K. Lele, A Modied Articial Viscosity Approach for Compressible Turbulence Simulations, J. Comput. Phys., to appear.

[4] D. W. Bogdanoff, Compressibility Effects in Turbulent Shear Layers, AIAA J., 21 (1983) 926-927.

[5] N. Chinzei, G. Masua, T. Komuro, A. Murakami, K. Kudou, Spreading of Two-Stream Supersonic Turbulent Mixing Layers, Phys. Fluids 29 (1986) 1345-1347.

[6] F. Ducros, F. Laporte, T. Soulères, V. Guinot, P. Moinat, and B. Caruelle, High-order Fluxes for Conservative Skew-Symmetric-like Schemes in Structured Meshes: Application to Compressible Flows, J. Comput. Phys., 161 (2000) 114-139.

[7] F. Ducros, V. Ferrand, F. Nicoud, C. Weber, D. Darracq, C. Gacherieu, and T. Poinsot, Large-Eddy Simulation of the Shock/ Turbulence Interaction, J. Comput. Phys., 152 (1999) 517-549.

[8] M. Farge, G. Pellegrino and K. Schneider Coherent Vortex Extraction in 3D Turbulent Flows using Orthogonal Wavelets, Phys. Rev. Lett., 5 (2001) 45011-45014.

[9] H. Foysi, S. Sarkar, The Compressible Mixing Layer: an LES Study, Theor. Comp. Fluid Dyn., DOI: 10.1007/s00162-009-0176-8 (2010).

[10] D.F. Griffiths, A.M. Stuart, and H.C. Yee, Numerical Wave Propagation in Hyperbolic Problems With Nonlinear Source Terms, SIAM J. Numer. Anal., 29 (1992) 1244-1260.

[11] M.R. Gruber, N.L. Messersmith and J.C. Dutton, Three-Dimensional Velocity Field in a Compressible Mixing Layer, AIAA Journal, 31 (1993) 2061-2067.

[12] A. Hadjadj and A. Kudryavtsev, Computation and Flow Visualization in High Speed Aerodynamics, J. Turbul., 6 (2005) 33-81.

[13] A. Hadjadj, H.C. Yee and B. Sjögreen, LES of Temporally Evolving Mixing Layer by High Order Filter Schemes, submitted to J. Comput. Phys.

[14] A. Harten, ENO Schemes with Subcell Resolution, J. Comput. Phys., 83 (1989) 148184.

[15] A.H. Honein and P. Moin, Higher Entropy Conservation and Numerical Stability of Compressible Turbulence Simulations, J. Comput. Phys., 201 (2004) 531-545.

[16] G.-S. Jiang and C.-W. Shu, Efficient Implementation of Weighted ENO Schemes, J. Comput. Phys., 126 (1996) 202-228. 
[17] E. Johnsen, J. Larson, A. V. Bhagatwala, W. H. Cabot, P. Moin, B. J. Olson, P. S. Rawat, S. K. Shankar, B. Sjögreen, H. C. Yee, X. Zhong, S. K. Lele, Assessment of High-Resolution Methods for Numerical Simulations of Compressible Turbulence with Shock Waves, J. Comput. Phys. 229 (2010) 1213-1237.

[18] M . Klein, A. Sadiki, and J. Janicka, A Digital Filter Based Generation of Inflow Data for Spatially Developing Direct Numerical or Large Eddy Simulation, J. Comp. Phys., 186 (2003) 652-665.

[19] D. Kotov, H.C. Yee, B. Sjögreen, W. Wang and C.W. Shu, Performance of Four High Oder Shock-Capturing Schemes for Stiff Source Terms with Discontinuities: Preliminary Results, CTR Annual Technical Brief, Stanford University, October, 2011.

[20] A. Lafon and H.C. Yee, Dynamical Approach Study of Spurious Steady-State Numerical Solutions for Nonlinear Differential Equations, Part III: The Effects of Nonlinear Source Terms in Reaction-Convection Equations, Comput. Fluid Dyn., 6 (1996) 1-36.

[21] A. Lafon and H.C. Yee, Dynamical Approach Study of Spurious Steady-State Numerical Solutions of Nonlinear Differential Equations, Part IV: Stability vs. Numerical Treatment of Nonlinear Source Terms, Comput. Fluid Dyn., 6 (1996) 89-123.

[22] R.J. LeVeque and H.C. Yee, A Study of Numerical Methods for Hyperbolic Conservation Laws with Stiff Source Terms, J. Comput. Phys., 86 (1990) 187-210.

[23] X.-S Li and C.-W. Gu, An All-Speed Roe-type Scheme and its Asymptotic Analysis of Low Mach Number Behaviour, J. Comput. Phys., 227 (2008) 5144-5159.

[24] S.-C. Lo, G.A. Blaisdell and A.S. Lyrintzis, High-order Shock Capturing Schemes for Turbulence Calculations, Int. J. Numer. Meth. Fluids 62 (2010) 473-498.

[25] P. Olsson and J. Oliger, Energy and Maximum Norm Estimates for Nonlinear Conservation Laws, RIACS Technical Report, 94.01 (1994).

[26] I. Mahle, J. Sesterhenn and R. Friedrich, Turbulent Mixing in Temporal Compressible Shear Layers Involving Detailed Diffusion Processes, J.Turb., 8 (2007) 1-2.

[27] P. Moin, K. Squires, W. Cabot and S. Lee, A Dynamic Subgrid Scale Model for Compressible Turbulence and Scalar Transport, Phys. Fluids 3 (1991) 2746.

[28] C. Pantano and S. Sarkar, A Study of Compressible Effects in the High-Speed Turbulent Shear Layer Using Direct Simulation, J. Fluid Mech., 451 (2002) 329-371.

[29] D. Papamoschou, A. Roshko, The Compressible Turbulent Shear Layer: An Experimental Study, J. Fluid Mech., 197 (1988) 453-477.

[30] P.L. Roe, Approximate Riemann Solvers, Parameter Vectors, and Difference Schemes, J. Comput. Phys., 43 (1981) 357-372. 
[31] M. M. Rogers and R. D. Moser, Direct Simulation of a Self-Similar Turbulent Mixing Layer, Phys. Fluids, 6 (1994) 903-923.

[32] M. Samimy, G.S. Elliot, Effect of compressibility on the characteristics of free shear layer, AIAA J., 28(3) (1990) 439-445.

[33] M. Samimy, M.F. Reeder, G.S. Elliott, Compressibility effects on large structures in free shear flows, Phys. Fluids, 4 (1992) 1251-1258.

[34] B. Sjögreen and H. C. Yee, Multiresolution Wavelet Based Adaptive Numerical Dissipation Control for Shock-Turbulence Computation, RIACS Technical Report TR01.01, NASA Ames Research Center (Oct 2000); also, J. Sci. Computing, 20 (2004) 211-255.

[35] B. Sjögreen and H.C. Yee, On Skew-Symmetric Splitting and Entropy Conservation Schemes for the Euler Equations, Proceedings of the 8th European Conference on Numerical Mathematics \& Advanced Applications (ENUMATH 2009), Uppsala University, June 29 - July 2, 2009, Uppsala, Sweden.

[36] B. Sjögreen, H.C.Yee, M.J.Djomehri, A.Lazanoff, and W.D.Henshaw, Parallel Performance of ADPDIS3D - A High Order Multiblock Overlapping Grid Solver for Hypersonic Turbulence, Proceedings of the 21st International Conference on Parallel CFD, Moffett Field, CA, May 18-22, 2009.

[37] B.Sjögreen and H.C.Yee, Variable High Order Multiblock Overlapping Grid Methods for Mixed Steady and Unsteady Multiscale Viscous Flows, Commun. Comput. Phys., 5 (2009), pp. 730-744.

[38] A. Stuart and A.R. Humphries Dynamical Systems and Numerical Analysis, Cambridge Monographs on Applied and Computational Mathematics, 1998.

[39] W. Wang, H.C. Yee, B. Sjögreen, T. Magin, and C.W. Shu, Construction of Low Dissipative High-Order Well-Balanced Filter Schemes for Nonequilibrium Flows, J. Comput. Phys., 230 (2011), pp 4316-4335. (doi:10.1016/j.jcp.2010.04.033, 2010).

[40] W. Wang, C.W. Shu, H.C. Yee and B. Sjögreen, High Order Finite Difference Methods with Subcell Resolution for Hyperbolic Conservation Laws with Stiff reaction Terms: Preliminary Results, Annual Research Briefs, Center for Turbulence Research, Stanford University (2010), PP. 149-160.

[41] H.C. Yee, P.K. Sweby, and D.F. Griffiths, Dynamical Approach Study of Spurious Steady-State Numerical Solutions for Nonlinear Differential Equations, Part I: The Dynamics of Time Discretizations and Its Implications for Algorithm Development in Computational Fluid Dynamics, NASA TM-102820, April 1990; J. Comput. Phys., 97 (1991) 249-310. 
[42] H.C. Yee, J.R. Torczynski, S.A. Morton, M.R. Visbal, and P.K. Sweby, On Spurious Behavior of CFD Simulations, AIAA 97-1869, Proceedings of the 13th AIAA Computational Fluid Dynamics Conference, June 29 - July 2, 1997, Snowmass, CO.; also Int. J. Num. Meth. Fluids, 30 (1999) 675-711.

[43] H.C. Yee and P.K. Sweby, Dynamics of Numerics \& Spurious Behaviors in CFD Computations, Keynote paper, 7th ISCFD Conference, Sept. 15-19, 1997, Beijing, China, RIACS Technical Report 97.06, June 1997.

[44] H.C. Yee, N.D. Sandham, and M.J. Djomehri, Low Dissipative High Order ShockCapturing Methods Using Characteristic-Based Filters, J. Comput. Phys., 150 (1999) 199-238.

[45] H.C. Yee, M. Vinokur, and M.J. Djomehri, Entropy Splitting and Numerical Dissipation, J. Comput. Phys., 162 (2000) 33-81.

[46] H.C. Yee and B. Sjögreen, Designing Adaptive Low Dissipative High Order Schemes for Long-Time Integrations, In Turbulent Flow Computation, (Eds. D. Drikakis \& B. Geurts), Kluwer Academic Publisher (2002); also RIACS Technical Report TR01-28, Dec. 2001.

[47] H.C. Yee, Building Blocks for Reliable Complex Nonlinear Numerical Simulations, In Turbulent Flow Computation, (Eds. D. Drikakis \& B. Geurts), Kluwer Academic Publisher (2002); also RIACS Technical Report TR01-28, Dec. 2001.

[48] H.C.Yee and B.Sjögreen, Efficient Low Dissipative High Order Schemes for Multiscale MHD Flows, II: Minimization of $\operatorname{div}(B)$ Numerical Error, J. Sci. Comp., 29 (2006) 115-164.

[49] H.C. Yee and B. Sjögreen, Development of Low Dissipative High Order Filter Schemes for Multiscale Navier-Stokes/MHD Systems, J. Comput. Phys., 225 (2007) 910-934.

[50] H. C. Yee, B. Sjögreen, and M. Barone, High order numerical schemes for hypersonic flow simulations, VKI Lecture Series. Course on Hypersonic Entry and Cruise Vehicles, 30 June - 3 July 2008.

[51] H.C. Yee and B. Sjögreen, High Order Filter Methods for Wide Range of Compressible Flow Speeds, Proceedings of ICOSAHOM 09 (International Conference on Spectral and High Order Methods). June 22-26, 2009, Trondheim, Norway.

[52] H.C. Yee, B. Sjögreen and A. Hadjadj, Comparative Study of High Order Schemes for LES of Temporal-Evolving Mixing Layers, Proceedings of ASTRONUM-2010, June 13-18, 2010, San Diego, Calif. 
[53] H.C. Yee, B. Sjögreen and A. Hadjadj, Local Flow Sensors in Controlling Numerical Dissipations for a Wide Spectrum of Flow Speed and Shock Strength, in preparation.

[54] H.C. Yee, B. Sjögreen, and A. Hadjadj, Comparative study of high order schemes for LES of temporal-evolving mixing layers, Proceedings of ASTRONUM-2010, June 13-18, 2010, San Diego, Calif;

[55] H.C. Yee, B. Sjögreen, C.W. Shu, W. Wang, T. Magin and A. Hadjadj, On Numerical Methods for Hypersonic Turbulent Flows, Proceedings of ESA 7th Aerothermodynamics Symposium, 9 - 12 May 2011 Site Oud Sint-Jan, Brugge, BelgiumASTRONUM-2010, June 13-18, 2010.

[56] H.C. Yee, D. Kotov, B. Sjögreen, W. Wang and C.W. Shu, Numerical Dissipation and Wrong Propagation Speed of Discontinuities For Stiff Source Terms, Proceedings of the ASTRONUM-2011, Valencia, Spain, June 13-17, 2011. 